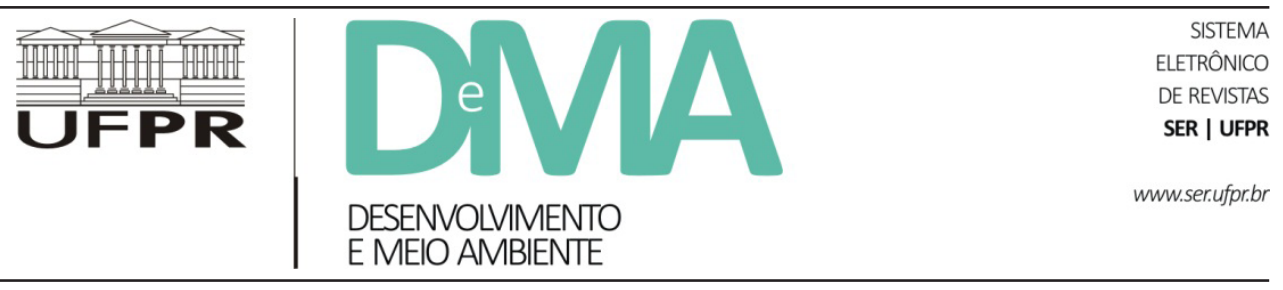

\title{
O impacto da Green Supply Chain Management no comportamento dos custos das empresas do setor energético listadas na BM\&FBOVESPA
}

\section{The Impact of the Green Supply Chain Management in the Costs Behavior of Companies in the Energy Sector Listed on BM\&FBOVESPA}

\author{
Emanuele ENGELAGE ${ }^{1 *}$, Altair BORGERT ${ }^{1}$ \\ ${ }^{1}$ Universidade Federal de Santa Catarina (UFSC), Florianópolis, SC, Brasil. \\ *E-mail de contato: manuengelage@hotmail.com
}

Artigo recebido em 13 de maio de 2016, versão final aceita em 30 de novembro de 2016.

RESUMO: Este estudo busca identificar qual o impacto das práticas da Green Supply Chain Management (GSCM) no comportamento dos custos de empresas do setor energético listadas na BM\&FBOVESPA, ao adotar como proxy de mensuração a certificação ISO 14001, a listagem do Índice de Sustentabilidade Empresarial (ISE) e a Lei ${ }^{\circ}$ 9.991/2000. Para tanto, analisam-se, por meio de modelos de regressão para dados em painel, as informações financeiras não consolidadas de 1995 a 2014, coletadas na Economática ${ }^{\circledR}$ e atualizadas pelo índice IPCA/IBGE. Os resultados divergem das ideias dos autores trazidos em discussão, à medida que as variáveis explicativas não se apresentam estatisticamente significantes, ao considerar os níveis usuais de intervalo de confiança. Assim, não é possível inferir que houve impacto na eficiência produtiva das empresas, com aumento ou diminuição da proporção do custo em relação às receitas de venda. Dessa forma, e ao considerar que esse efeito pode ser compensado por outros fatores, busca-se analisar isoladamente os custos e as receitas. Os resultados demonstram que todas as variáveis explicativas não são estatisticamente significativas para as análises de receitas, mas, para as análises de custos, tem-se que, quanto maior o nível de adesão às práticas de GSCM, maiores são os custos dos produtos vendidos.

Palavras-chave: Green Supply Chain Management; comportamento dos custos; práticas sustentáveis.

ABSTRACT: This study seeks to identify the impact of the practices of Green Supply Chain Management (GSCM) in the costs behavior of companies in the energy sector listed on BM\&FBOVESPA, by adopting as measurement proxy the ISO 14001 certification, the Managerial Sustainability Index (ISE) listing, and the 9.991/2000 Law. Therefore, we analyzed, by regression models for panel data, the unconsolidated financial statements from 1995 to 2014, collected in Economática ${ }^{\circledR}$ and updated by the IPCA/IBGE index. The results differ from the ideas brought by the authors in question, as the explicative variables are not statistically significant when considering the usual confidence interval levels. Thus, it is not possible to infer that there was an impact on the productive efficiency 
of the companies, with an increase or decrease in the proportion of the cost in relation to sales revenues. This way, and considering that this effect may be offset by other factors, it seeks to separately analyze the costs and revenues. The results show that all the explicative variables are not statistically significant for the analysis of revenues, but, for the cost analysis, they show that the higher the level of adherence to GSCM practices, the greater the cost of goods sold.

Keywords: Green Supply Chain Management; cost behavior; sustainable practices.

\section{Introdução}

A adoção de práticas sustentáveis pelas organizações tem se intensificado nos últimos anos em decorrência da elevação da conscientização ambiental por parte da sociedade (Chien \& Shih, 2007; Ribeiro \& Santos, 2013). Diante dos impactos oriundos de ações desordenadas, como a exploração de recursos naturais finitos e a degradação ambiental, a sociedade exerce pressão, por meio da priorização de produtos e empresas com atitudes ecologicamente corretas, para que as companhias busquem um novo conceito de desenvolvimento, com a adoção de políticas de controle, preservação e recuperação ambiental (Campos et al., 2009; Ribeiro \& Santos, 2013; Srisorn, 2013).

A introdução de iniciativas verdes por parte das organizações deriva não apenas da pressão dos stakeholders, mas também das exigências do mercado, da imposição legislativa e da concorrência cada vez mais acirrada, que faz com que as empresas busquem se desenvolver sustentavelmente e, consequentemente, melhorar sua imagem e tornar-se mais inovadoras e criativas (Ribeiro \& Santos, 2013; Seroka-Stolka, 2014).

Salienta-se que o desenvolvimento sustentável é aquele que satisfaz as necessidades presentes sem comprometer a capacidade das gerações futuras de suprir suas próprias necessidades (ONU, 1991). Dessa forma, atitudes sustentáveis envolvem não só responsabilidade ambiental, mas a integração desta às ações voltadas à economia e à sociedade, em que se busca um constante equilíbrio entre redução de custos, atendimento às metas de lucros, diminuição na geração de poluentes e resíduos, minimização de desperdícios e diminuição na utilização de recursos (Arantes et al., 2014).

Uma abordagem interdisciplinar utilizada para designar a incorporação de práticas sustentáveis desde a extração de matérias-primas até a destinação final dos produtos, que completa o ciclo produção/consumo/pós-uso, é a Green Supply Chain Management (GSCM). Essa sistemática inclui desde o monitoramento relativo a programas de gestão ambiental, como regulamentações e conformidades, até práticas mais proativas, como a minimização da utilização de recursos, reciclagem, recuperação e reconstrução, ao combinar fatores como compras ecológicas, fabricação verde, gestão de materiais, distribuição verde, marketing e logística reversa (Chien \& Shih, 2007; Seman et al., 2012; Lopes et al., 2013; Arantes et al., 2014).

A identificação e a mensuração de práticas sustentáveis ao longo da cadeia de suprimentos podem se dar de diferentes maneiras. Alguns dos indicadores de adesão a essas práticas pelas organizações, apresentados por estudos vinculados à temática, são: (i) a NBR ISO 14001, que desenvolve padrões e normatizações baseadas nas necessidades das empresas, setores e partes interessadas em âmbito mundial, com o objetivo de direcionar as empresas à busca pela minimização dos efeitos de suas atividades no ambiente, bem como à melhoria contínua de seu desempenho (Raines; 2002; Ba- 
bakri et al., 2003; Ann et al., 2006; Campos et al., 2009; Ferron et al., 2012; Lopes et al., 2013; Zaro et al., 2015); e (ii) o Índice de Sustentabilidade Empresarial (ISE), que visa demonstrar a eficiência econômica, o equilíbrio ambiental, a justiça social e a governança corporativa de empresas e grupos listados na BM\&FBOVESPA e comprometidos com a sustentabilidade (Rover et al., 2008; Da Costa et al., 2009; BM\&FBOVESPA, 2016).

Destaca-se que a décima carteira do ISE, divulgada em 2015 e que vigorou até 2 de janeiro de 2016, reúne 51 ações de 40 companhias de 19 setores diferentes e soma $\mathrm{R} \$ 1,22$ trilhão em valor de mercado, o equivalente a $49,87 \%$ do total do valor das companhias com ações negociadas na BM\&FBOVESPA, com base no fechamento de $24 / 11 / 2014$. Destas, nove companhias classificam-se no setor de utilidade pública com fornecimento de energia elétrica, o qual exerce maior representatividade perante os setores da listagem $(22,5 \%)$ (BM\&FBOVESPA, 2014).

Isso posto, este estudo direciona suas análises ao segmento de energia elétrica e, portanto, inclui mais uma variável para mensuração de práticas sustentáveis no decorrer da cadeia de suprimentos, haja vista que esse setor em particular enquadra-se na Lei $n^{\circ} 9.991 / 2000$, que obriga as companhias a destinarem o percentual mínimo de $0,5 \%$ de sua receita líquida de vendas para atitudes voltadas a evitar ou reduzir o desperdício energético, o que constitui mais um indício da adoção de práticas sustentáveis (Brasil, 2000).

Contudo, mais do que possuir esses indicadores (ISO 14001, carteira ISE e atendimento à Lei $\mathrm{n}^{\mathrm{o}}$ 9.991/2000), as empresas necessitam associar à temática ambiental o desenvolvimento econômico, a fim de manter-se competitivas e atuantes no mercado. A redução de custos é um objetivo importante para aquelas que buscam melhoria de desempenho e constitui uma das suas principais dimensões estratégicas, principalmente para as que atuam no comércio internacional. Dessa forma, torna-se necessário compreender como os custos se comportam em função de variações nos níveis de atividades, volume de vendas e estrutura operacional das empresas, ao considerar as interferências ambientais, sociais e econômicas (Barbieri, 1998; Richartz, 2013).

No que se refere ao custo de implantação e manutenção de práticas sustentáveis em relação às receitas auferidas no decorrer da cadeia de suprimentos, há divergências de opiniões entre autores: uns alegam a ocorrência de perdas e desvantagens, e outros alegam ganhos financeiros com a adesão de práticas ambientalmente amigáveis. Entre os autores que alegam perdas financeiras com elevação de custos, ao tomar por bases diferentes proxies de mensuração, estão: Walley \& Whitehead (1994); Babakri et al. (2003); Oliveira \& Serra (2010); Luthra et al. (2011); Abbasi \& Nilsson (2012) e Zaro et al. (2015). Por outro lado, alguns dos autores que alegam que as práticas de GSCM acarretam ganhos financeiros, seja pela redução de custos, seja pela elevação proporcionalmente maior da receita, são: Porter \& Van der Linde (1995); Raines (2002); Rao \& Holt (2005); Ann et al. (2006); Alberton \& Costa Jr. (2007); Chien \& Shih (2007); Ambec \& Lanoie (2008); Campos et al. (2009); Yang et al. (2010); Ferron et al. (2012); Pak (2013); e Jong et al. (2014).

Assim, ao se conjecturar a adoção da GSCM como um diferencial competitivo, torna-se importante a compreensão do comportamento dos custos, ao considerar também fatores ambientais e sociais, haja vista que esse entendimento oportuniza o fornecimento de informações detalhadas que auxiliam a gestão empresarial na projeção das atividades, no planejamento e na tomada de decisão estratégica 
(Zaro et al., 2015). Diante do exposto, este estudo pretende responder à seguinte pergunta de pesquisa: qual o impacto da Green Supply Chain Management no comportamento dos custos das empresas do setor energético listadas na BM\&FBOVESPA?

Portanto, o objetivo geral deste estudo é identificar qual a influência das práticas da GSCM no comportamento dos custos de empresas do setor energético listadas na BM\&FBOVESPA. Para tanto, tomam-se por base como fator de medição para essas práticas a certificação ISO 14001, a listagem do Índice de Sustentabilidade Empresarial (ISE) e a Lei ${ }^{\circ}$ 9.991/2000.

Raines (2002), ao realizar um estudo a fim de verificar a percepção dos gestores em relação à ISO 14001 em âmbito mundial, identificou, como uma das vantagens da certificação, a melhora nos resultados financeiros. Esse ganho, segundo o autor, se potencializa nos países em desenvolvimento, onde as empresas acabam por obter visibilidade e preferências no mercado. Nesse sentido, busca-se verificar se essa vantagem financeira também ocorre no Brasil ao se utilizar como parâmetro o setor energético, com o diferencial de estender-se às práticas de GSCM como um todo.

\section{Referencial teórico}

As pressões por parte do governo e da sociedade, motivadas principalmente pela crescente deterioração do ambiente, têm dado destaque à GSCM entre os pesquisadores e praticantes de operações da cadeia de suprimentos, uma vez que esta promove a eficiência e a sinergia entre os parceiros do negócio e contribui para o aumento da performance ambiental, social e econômica, ao minimizar desperdícios e auxiliar na economia de custos (Rao \& Holt, 2005; Srivastava, 2007; Lopes et al., 2013).
A definição de GSCM foi apresentada pela primeira vez em 1996 pela Manufacturing Research Association (MRC), e considera a influência do meio ambiente para a otimização de recursos (Chunguang et al., 2008).

Problemas de desempenho ambiental ou social podem afetar a marca e as negociações das ações de uma empresa. Assim, a GSCM se remete a questões de cunho socioambiental integradas ao enfoque competitivo, pois trata do processo de planejamento, implementação e controle eficiente na busca pela elevação dos lucros durante todo o ciclo de vida de bens e produtos. Essa busca se dá através da interação de programas de sustentabilidade e práticas proativas que potencializam o uso de recursos, diminuem a poluição e agreguem valor à empresa. Dentre essas ações, pode-se citar a introdução da sistemática dos vários "Rs", ou seja, práticas de redução, reúso, recondicionamento, reciclagem, remanufatura e logística reversa (Rogers \& Tibben-Lembke, 1999; Srivastava, 2007; Seuring et al., 2008; Lopes et al., 2013).

Contudo, mensurar o nível de desenvolvimento sustentável de uma organização ao longo da cadeia de suprimentos não é uma tarefa simples e, na literatura, encontram-se diferentes abordagens para essa delimitação. Uma dessas abordagens refere-se ao indicador ISO 14001, que é uma norma de especificação e, portanto, de certificação da responsabilidade social e ambiental das entidades credenciadas, ao considerar uma descrição detalhada de diretrizes e requisitos dos processos e produtos, que são auditados com o intuito de se verificar a conformidade com a norma. Essa ferramenta propicia às organizações a criação de mecanismos estruturados para melhoria contínua do desempenho ambiental com base no ciclo PDCA, ou seja, planejar, executar, verificar e agir (Barbieri, 1998; ISO, 2016; Oliveira \& Serra, 2010; Zaro et al., 2015). 
Outra abordagem utilizada, mais especificamente no Brasil, que constitui indício de sustentabilidade pelas organizações é a listagem do ISE, calculado pela BM\&FBOVESPA. Ele foi criado em dezembro de 2005 como uma iniciativa pioneira na América Latina, uma vez que o Brasil foi o quarto país no mundo a desenvolver um indicador com o objetivo de demonstrar o desempenho de mercado de empresas que adotam princípios de gestão sustentável (Marcondes \& Bacarji, 2010). Esse indicador visa induzir boas práticas no meio empresarial brasileiro, ao se tornar referência para investimentos responsáveis, pois busca criar um ambiente compatível com as demandas de desenvolvimento sustentável da sociedade contemporânea e estimular o compromisso ético das corporações (BM\&FBOVESPA, 2016).

A carteira ISE publicada em 2014, válida de 5 de janeiro de 2015 até 2 de janeiro de 2016, apresenta 40 companhias de 19 setores distintos, consideradas com melhores práticas de sustentabilidade e que se diferenciam em termos de qualidade, nível de compromisso com o desenvolvimento sustentável, equidade, transparência, prestação de contas e natureza do produto, além do desempenho empresarial nas dimensões econômico-financeira, social, ambiental e de mudanças climáticas (BM\&FBOVESPA, 2016). Do total de empresas e setores dessa listagem, nove correspondem ao segmento de energia elétrica, o qual possui maior representatividade.

Ressalta-se que o setor energético, objeto deste estudo, possui mais um diferencial no que diz respeito à sustentabilidade. Em 2000, por meio do Programa de Eficiência Energética das Empresas de Distribuição (PEE), regulamentado pela Lei $\mathrm{n}^{\circ}$ 9.991/2000, a Agência Nacional de Energia Elétrica (ANEEL) estipulou que as empresas concessionárias de distribuição de energia devem destinar o percentual mínimo de $0,5 \%$ de sua receita líquida de vendas às atitudes voltadas para a redução e o combate do desperdício energético (Brasil, 2000).

Para que as empresas continuem competitivas, mesmo com a adequação ou implantação de práticas sustentáveis no decorrer da cadeia de suprimentos, seja por qualquer um desses mecanismos, é importante conhecer os impactos financeiros de sua adoção. Dessa forma, a análise do comportamento de custos, de forma temporal ou comparativa a outras empresas, serve como parâmetro para delimitar como a GSCM afeta financeiramente as organizações.

A importância de se investigar o comportamento dos custos apoia-se na melhoria de condições para prever situações e planejar as atividades, uma vez que possibilita manter o controle dos processos e realizar predições de tendências para maximização dos lucros, melhoria contínua e aumento do nível competitivo. A análise de custos históricos, bem como o entendimento de como estes se portam mediante situações específicas, significa compreender a interação dos diversos direcionadores de custos e utilizar essa informação a favor da gestão, na predição de resultados e na tomada de decisão estratégica (Melvin, 1988; Shank \& Govindarajan, 1997; Richartz, 2013)

No que tange ao comportamento dos custos em relação à incorporação de práticas sustentáveis, Zaro et al. (2015), ao realizar um estudo no intuito de identificar qual o impacto da certificação ISO 14001 nos custos das empresas do setor petroquímico listadas na BM\&FBOVESPA, sugerem que a eficiência produtiva das empresas estudadas diminuiu, devido ao fato de o custo dos produtos vendidos ter aumentado mais do que as receitas líquidas de vendas.

Nessa mesma direção, Babakri et al. (2003) e Oliveira \& Serra (2010) identificaram que os custos empresariais se elevam com a adoção de 
condutas voltadas à obtenção da certificação ISO 14001. Contudo, utilizaram abordagens de pesquisa diferenciadas, cujo direcionamento está em fatores críticos gerais e na análise dos benefícios e dificuldades de implantação da certificação, e não exclusivamente nos custos.

Luthra et al. (2011) realizaram um estudo com o objetivo de desenvolver um modelo estrutural das barreiras na implantação da GSCM em indústrias automobilísticas indianas e encontrar relações contextuais entre elas, em que uma das dificuldades verificadas baseia-se exatamente nas implicações de custo. Abbasi \& Nilsson (2012) e Walley \& Whitehead (1994) corroboram a ideia de elevação de custos ao referir-se à GSCM como um todo, porém seus estudos possuem diferentes diretrizes. Salienta-se que Abbasi \& Nilsson (2012), ao explorar na literatura temas e desafios da GSCM, também citam a complexidade operacional, as mudanças na mentalidade e na cultura e as incertezas do processo.

Em alternativa aos estudos anteriores, Lamming \& Hampson (1996) apontam que tanto o aumento como a redução dos custos ocorrem em diferentes momentos da adoção de práticas sustentáveis. Na implantação dos sistemas de gestão sustentável, as empresas acabam por aumentar seus custos, devido aos investimentos realizados, porém, ao possuírem elevado desempenho ambiental, podem diminuir ou eliminar perdas e desperdícios.

Dessa forma, há estudos que reforçam a ideia de "ganha-ganha", defendida por Porter \& Van der Linde (1995), a qual argumenta que a melhoria do desempenho ambiental de uma empresa pode levar ao melhor desempenho econômico ou financeiro, e não necessariamente a um aumento no custo (Teoria de Porter). Assim, Ambec \& Lanoie (2008) realizaram uma pesquisa que revisa evidências empíricas de melhoria em termos de desempenho ambiental, econômico e financeiro, a fim de traçar um diagnóstico do tipo de empresas com maior probabilidade de colher maiores benefícios com a adesão às práticas sustentáveis. $\mathrm{O}$ estudo revelou uma potencial redução de custos para as empresas com melhor desempenho ambiental, com melhoria no gerenciamento de riscos e na relação com os stakeholders, nos custos de capital e nos custos com mão de obra, materiais, energia e serviços.

O estudo de Yang et al. (2010) analisou as inter-relações entre programas de melhoria contínua, gestão de fornecedores e gestão ambiental. Para tal, os autores aplicaram um questionário em empresas do setor elétrico e eletrônico de Taiwan e da China e construíram um modelo de regressão múltipla, em que os resultados estatísticos sugerem que as empresas com gestão ambiental proativa aumentam suas vantagens competitivas, por meio da redução de custos e da inovação nos processos, pois criam parcerias mais estreitas com fornecedores $\mathrm{e}$ estabelecem práticas sólidas de melhoria contínua.

Rao \& Holt (2005) pesquisaram a ligação entre a GSCM e o aumento da competitividade e da competência econômica de uma amostra de organizações do Sudeste Asiático e apontam como fatores positivos a redução de custos operacionais, a integração de fornecedores ao processo de tomada de decisão, estratégias de compras diferenciadas, redução de desperdícios, substituição de materiais e matérias-primas, redução nas emissões de gases de efeito estufa, melhor utilização de recursos naturais, desenvolvimento mais eficiente de novos produtos, inovação, entre outros. Chien \& Shih (2007) e Pack (2013) também argumentam sobre a redução de custos com a GSCM. No entanto, suas pesquisas direcionam-se respectivamente à verificação de práticas que possam ser introduzidas e adaptadas e aos fatores que influenciam a sua adoção. 
Também, quanto à redução de custos, porém voltados especificamente à ISO 14001, têm-se os estudos de Raines (2002), Ann et al. (2006), Alberton \& Costa Jr. (2007), Campos et al. (2009), Ferron et al. (2012) e Jong et al. (2014), cujos objetivos, procedimentos metodológicos e enfoques gerais de pesquisa diferem-se entre si, mas ambos relatam melhoria nos resultados empresariais com a adoção de práticas que são requisitos para a certificação, seja pela redução dos custos, seja pelo aumento das receitas.

Destaca-se que as pesquisas de Ferron et al. (2012), Alberton \& Costa Jr. (2007) e Campos et al. (2009) foram realizadas no Brasil, e as três utilizaram indicadores financeiros como proxies de medição para o desempenho organizacional, a fim de analisar como estes são impactados pela certificação ISO 14001.

Diante das divergências encontradas na literatura, em que alguns autores alegam a elevação de resultados financeiros com a introdução de práticas da GSCM, e outros, a sua redução, faz-se necessário compreender o comportamento dos custos em face das receitas auferidas. Esse entendimento auxilia no dimensionamento dos impactos de práticas sustentáveis e possibilita a previsão de tendências, o que pode fazer com que os custos deixem de ser vistos como uma barreira para a incorporação da GSCM, conforme abordado por diversos autores (Walley \& Whitehead, 1994; Lamming \& Hampson, 1996; Babakri et al., 2003; Rao \& Holt, 2005; Ann et al., 2006; Luthra et al., 2011; Abbasi \& Nilsson, 2012; Seman et al., 2012).

\section{Procedimentos metodológicos}

Com o intuito de atingir o objetivo da pesquisa, este tópico esboça e esclarece os procedimentos metodológicos e, para tanto, subdivide-se em três seções, quais sejam: a coleta dos dados, a definição das variáveis e os procedimentos para a análise dos dados.

\subsection{Coleta de dados}

Para a realização desta pesquisa, selecionaram-se as empresas do subsetor de energia elétrica - inserido no setor de utilidade pública - cujas ações foram negociadas na BM\&FBOVESPA no período entre 1995 e 2014. A determinação do ponto de corte no ano de 1995 justifica-se por esse ser o primeiro ano completo após a adoção do Plano Real, quando o país atingiu certa estabilidade econômica em termos de inflação. Já o ano de 2014 foi estabelecido em função da disponibilidade de informações financeiras completas.

A definição do setor energético dá-se pela sua representatividade entre as empresas listadas na carteira ISE 2015 - nove entre as 40 empresas consideradas com melhores práticas sustentáveis $(22,50 \%)$. Destaca-se que se encontram listadas no setor de energia elétrica na BM\&FBOVESPA 66 empresas, porém, neste estudo, analisam-se apenas 56, uma vez que dez não apresentam informações financeiras consolidadas disponíveis na base de dados Economática ${ }^{\circledR}$. Portanto, dessas 56 empresas, foram coletadas para as análises as informações de receita líquida de vendas (RLV), custo dos produtos vendidos (CPV), despesas com vendas (DV), despesas administrativas (DA) e ativo total (AT), ajustados pelo índice de inflação medido pelo IPCA, com auxílio da própria base de dados.

Em função da variável ISO 14001, para identificar se há certificação ou não, analisam-se individualmente, em cada empresa, a data de obtenção e o período médio entre a implantação do sistema 
de gestão e a concessão do certificado. Para isso, tomam-se por base os dados extraídos dos relatórios de administração disponíveis no site da CVM ou da BM\&FBOVESPA, em que empresas que não apresentam informação de certificação nesses relatórios são consideradas como "não certificadas". Para a identificação da data de obtenção do certificado, observa-se a data da primeira certificação obtida pela empresa, uma vez que a ISO 14001 é concedida por unidade empresarial e os relatórios da administração são informações consolidadas. Destaca-se que, para as empresas pertencentes ao relatório ISE 2015 cuja informação de certificação não consta nos relatórios da administração, a pesquisa amplia-se para os sites oficiais.

\subsection{Definição das variáveis}

Com os dados coletados e com vistas à obtenção de respostas direcionadas ao problema de pesquisa, faz-se necessária a definição das variáveis em estudo. Para tanto, define-se como variável dependente, em uma primeira análise, a razão entre o custo dos produtos vendidos e a receita líquida de vendas (CPV/RLV), pois se busca uma medida de eficiência dos custos para verificar qual o seu comportamento em função da adoção de práticas de GSCM. Num segundo momento, ampliam-se as análises para a razão entre as despesas administrativas e de vendas e a receita líquida de vendas (|DAs + DVs//RLV), na tentativa de reduzir a influência de classificações distintas entre as organizações.

Como variáveis independentes ou explicativas, ou seja, aquelas que capturam o efeito da adoção das práticas de GSCM, são estabelecidos três níveis de análise, que representam, respectivamente, os níveis inferior, intermediário e superior de adoção, quais sejam: classe 1 - sem certificação
ISO 14001; classe 2 - com certificação ISO 14001; e classe 3 - listadas no relatório ISE, que inclui empresas com e sem certificação. Destaca-se que essa metodologia de divisão por níveis de práticas sustentáveis também é utilizada por Alberton \& Costa Jr. (2007) e Campos et al. (2009).

Por fim, também como variável explicativa, utiliza-se a Lei $n^{\circ}$ 9.991/2000, uma vez que esta pode impactar a intensidade de práticas sustentáveis das organizações, e o porte da empresa é utilizado como uma variável de controle.

\subsection{Instrumentos e técnicas para análise dos dados}

Após a obtenção dos dados e a definição das variáveis utilizadas, exportam-se as informações para planilhas do software Microsoft Excel ${ }^{\circledR}$ para tabulação e organização e, com apoio do software estatístico Stata ${ }^{\circledR}$ SR for Windows ${ }^{\circledR}$ versão 12 , analisam-se as observações fornecidas, para estabelecer a influência de práticas de GSCM no comportamento dos custos organizacionais.

Para as análises, utiliza-se a técnica de regressão para dados em painel, uma vez que se busca evidenciar a heterogeneidade individual da amostra ao analisar conjuntamente as características de cross-section e séries de tempo, ou seja, analisar várias empresas em uma evolução temporal (Fávero, 2015). Outros autores que utilizaram como ferramenta de análise o modelo econométrico de regressão foram: Yang et al. (2010), Ferron et al. (2012) e Zaro et al. (2015). O primeiro utilizou o modelo de regressão múltipla, e os demais, a regressão para dados em painel. Desse modo, este estudo utiliza os seguintes modelos de regressão para as diferentes análises a serem realizadas: 
Análise 1:
(a) $\frac{C P V}{R L V}=\alpha+\beta 1 \cdot$ Classe $+\beta 2 \cdot$ Porte $+\varepsilon$,
(b) $\frac{C P V}{R L V}=\alpha+\beta 1 \cdot$ ISO $14001+\beta 2 \cdot$ Lei $+\beta 3 \cdot$ Porte $+\varepsilon$,

Análise 2:

(c) $\frac{(D A+D V)}{R L V}=\alpha+\beta 1 \cdot$ Classe $+\beta 2 \cdot$ Porte $+\varepsilon$,
(d) $\frac{(D A+D V)}{R L V}=\alpha+\beta 1 \cdot$ ISO $14001+\beta 2 \cdot$ Lei $+\beta 3 \cdot$ Porte $+\varepsilon$

Destaca-se que a análise 1 se refere aos dois modelos estabelecidos quando a variável dependente é a razão entre o CPV e a RLV, que representa uma medida de eficiência dos custos. Por sua vez, a análise 2 refere-se aos dois modelos cuja variável dependente é a razão entre as DAs e as DVs e a RLV.

As demais informações consideram as variáveis já apresentadas no tópico anterior e as intrínsecas ao modelo, em que: o símbolo $\alpha$ representa o intercepto; $\beta 1, \beta 2$ e $\beta 3$ são os coeficientes de cada variável; X1 do primeiro modelo de regressão, em ambas as análises (modelos "a" e "c"), refere-se aos três níveis de práticas de GSCM e, para captar o seu efeito, utilizam-se duas dummy. Ainda, na primeira regressão, adota-se a variável de controle porte, que é definida pelo AT de cada organização distribuído em quatro níveis distintos, que variam do menor (1) para o maior (4). Essa divisão baseia-se na determinação das medidas de dispersão, por meio da inclusão de variáveis dummy.

Como a variável classe, em seu último nível (classe 3), não apresenta diferenciação entre empresas certificadas e não certificadas, gera-se mais um modelo de regressão para ambas as análises (modelos "b" e "d"), que objetiva averiguar a variável ISO 14001 isoladamente. Além do mais, no modelo anterior só há análises a partir da data de início da divulgação do relatório ISE (dezembro de
2005), porém há empresas certificadas antes desse período, nas quais também se pretende analisar os efeitos sobre o comportamento dos custos. Nesse mesmo sentido, a variável Lei no 9.991 também é inclusa nesse último modelo, uma vez que foi criada em 2000 e o seu efeito não seria captado na regressão anterior. Salienta-se que ambas as variáveis explicativas dessa análise são inclusas no modelo em forma de dummy. Por fim, assim como no modelo anterior, neste também se utiliza o porte como variável de controle, seguindo, para tanto, as mesmas especificações e enquadramentos.

Para fins de consistência das análises, excluem-se as informações financeiras inexistentes, zeradas e consideradas discrepantes, por meio da verificação do boxplot (amplitude interquartis). Assim, obtêm-se 820 observações nos dois modelos da análise de custo em relação à receita e 667 observações nos dois modelos da análise de despesas. Em seguida, realizam-se os testes de confirmação da melhor estimação dos modelos lineares de regressão para dados em painel, a verificação de alguns pressupostos básicos que são intrínsecos ao modelo e, então, geram-se as análises.

\section{Apresentação e análise dos resultados}

Inicialmente, numa análise do conjunto de dados, à luz do modelo proposto, pode-se inferir que o painel é desbalanceado, haja vista que os períodos (anos) que dispõem de informação variam entre as empresas, ou seja, não há dados disponíveis para todas as empresas em todos os anos (Fávero, 2015).

Isso posto, realizam-se alguns testes para a definição da melhor estimação dos modelos lineares de regressão para dados em painel, e tem-se que o modelo mais adequado aos dados da análise 1 é o de efeito aleatório e, para os dados da análise 2, é o de efeito fixo, conforme pode-se perceber nos resultados da Tabela 1 . 
TABELA 1 - Testes para definição do melhor modelo de estimação

\begin{tabular}{lllll}
\hline & \multicolumn{1}{c}{ Hipótese nula } & \multicolumn{1}{c}{ Hipótese alternativa } & \multicolumn{1}{c}{ Análise 1 } & Análise 2 \\
\hline Teste de Chow & $\begin{array}{l}\text { Os interceptos são iguais } \\
\text { para todas as cross- } \\
\text { sections (POLS) }\end{array}$ & $\begin{array}{l}\text { Os interceptos são diferentes } \\
\text { para todas as cross-sections } \\
\text { (efeitos fixos) }\end{array}$ & $\begin{array}{l}\text { Rejeita } \mathrm{H}_{0} \text { a um nível de } \\
\text { significância de 5\% }\end{array}$ & $\begin{array}{l}\text { Rejeita } \mathrm{H}_{0} \text { a um nível de } \\
\text { significância de 5\% }\end{array}$ \\
\hline $\begin{array}{l}\text { Teste LM de } \\
\text { Breusch-Pagan }\end{array}$ & $\begin{array}{l}\text { A variância dos resíduos } \\
\text { que refletem a diferença } \\
\text { é igual a zero (POLS) }\end{array}$ & $\begin{array}{l}\text { A variância dos resíduos que } \\
\text { refletem a diferença é diferente } \\
\text { de zero (efeitos aleatórios) }\end{array}$ & $\begin{array}{l}\text { Rejeita } \mathrm{H}_{0} \text { a um nível de } \\
\text { significância de 5\% }\end{array}$ & $\begin{array}{l}\text { Rejeita } \mathrm{H}_{0} \text { a um nível de } \\
\text { significância de 5\% }\end{array}$ \\
\hline $\begin{array}{l}\text { Teste de } \\
\text { Hausman }\end{array}$ & $\begin{array}{l}\text { O modelo de correção de } \\
\text { erros é adequado (efeitos } \\
\text { aleatórios) }\end{array}$ & $\begin{array}{l}\text { O modelo de correção de erros } \\
\text { não é adequado (efeitos fixos) }\end{array}$ & $\begin{array}{l}\text { Não rejeita } \mathrm{H}_{0} \text { a um nível } \\
\text { de significância de 5\% }\end{array}$ & $\begin{array}{l}\text { Rejeita } \mathrm{H}_{0} \text { a um nível de } \\
\text { significância de 5\% }\end{array}$ \\
\hline
\end{tabular}

FONTE: Elaborada pelos autores

Também se faz necessária a confirmação de alguns pressupostos para a utilização do modelo de regressão, quais sejam: a não correlação dos resíduos com as variáveis independentes (homocedasticidade); a aleatoriedade e a independência dos resíduos (ausência de autocorrelação); a não correlação elevada entre as variáveis explicativas (ausência de multicolinearidade); e a distribuição normal dos resíduos (Fávero, 2015). Dessa forma, a Tabela 2 apresenta os resultados dos testes de cada pressuposto.
Conforme se pode percebe na Tabela 2, o teste Jarque-Bera rejeita a hipótese nula de distribuição normal dos resíduos para todos os modelos. Portanto, examinam-se individualmente os dados para verificar a sua distribuição, uma vez que, com um número elevado de observações e considerando-se o conceito de normalidade assintótica dos resíduos, os estimadores podem continuar consistentes e garantir a validade dos resultados, o que viabiliza a sua análise (Gujarati, 2006).

TABELA 2 - Testes dos pressupostos do modelo de regressão

\begin{tabular}{|c|c|c|c|c|}
\hline & Heterocedasticidade & Autocorrelação & Normalidade & Multicolinearidade \\
\hline Teste realizado & Breusch-Pagan e White & Wooldridge & Jarque-Bera & Fator de impacto da variância (VIF) \\
\hline Hipótese nula $\left(\mathrm{H}_{0}\right)$ & $\begin{array}{l}\text { Possui homocedasticidade } \\
\text { nos dados }\end{array}$ & $\begin{array}{l}\text { Ausência de correlação } \\
\text { nos dados }\end{array}$ & $\begin{array}{l}\text { Distribuição normal dos } \\
\text { resíduos }\end{array}$ & \multirow{2}{*}{$\begin{array}{l}\text { Se VIF }>10 \text {, diz-se que a variável é } \\
\text { altamente colinear (Gujarati, 2006) }\end{array}$} \\
\hline $\begin{array}{l}\text { Hipótese } \\
\text { alternativa }\left(\mathrm{H}_{1}\right)\end{array}$ & $\begin{array}{l}\text { Possui heterocedasticidade } \\
\text { nos dados }\end{array}$ & $\begin{array}{l}\text { Existência de } \\
\text { correlação nos dados }\end{array}$ & $\begin{array}{l}\text { Distribuição não normal } \\
\text { dos resíduos }\end{array}$ & \\
\hline $\begin{array}{l}\text { Resultado modelo } \\
\text { de regressão (b) }\end{array}$ & $\begin{array}{l}\text { Rejeita } \mathrm{H}_{0} \text { a um nível de } \\
\text { significância de } 5 \%\end{array}$ & $\begin{array}{l}\text { Rejeita } \mathrm{H}_{0} \text { a um nível de } \\
\text { significância de } 5 \%\end{array}$ & $\begin{array}{l}\text { Rejeita } \mathrm{H}_{0} \text { a um nível de } \\
\text { significância de } 5 \%\end{array}$ & Não há multicolinearidade \\
\hline $\begin{array}{l}\text { Resultado modelo } \\
\text { de regressão (c) }\end{array}$ & $\begin{array}{l}\text { Rejeita } \mathrm{H}_{0} \text { a um nível de } \\
\text { significância de } 5 \%\end{array}$ & $\begin{array}{l}\text { Rejeita } \mathrm{H}_{0} \text { a um nível } \\
\text { de significância de } 5 \%\end{array}$ & $\begin{array}{l}\text { Rejeita } \mathrm{H}_{0} \text { a um nível de } \\
\text { significância de } 5 \%\end{array}$ & Não há multicolinearidade \\
\hline
\end{tabular}

FONTE: Elaborada pelos autores 


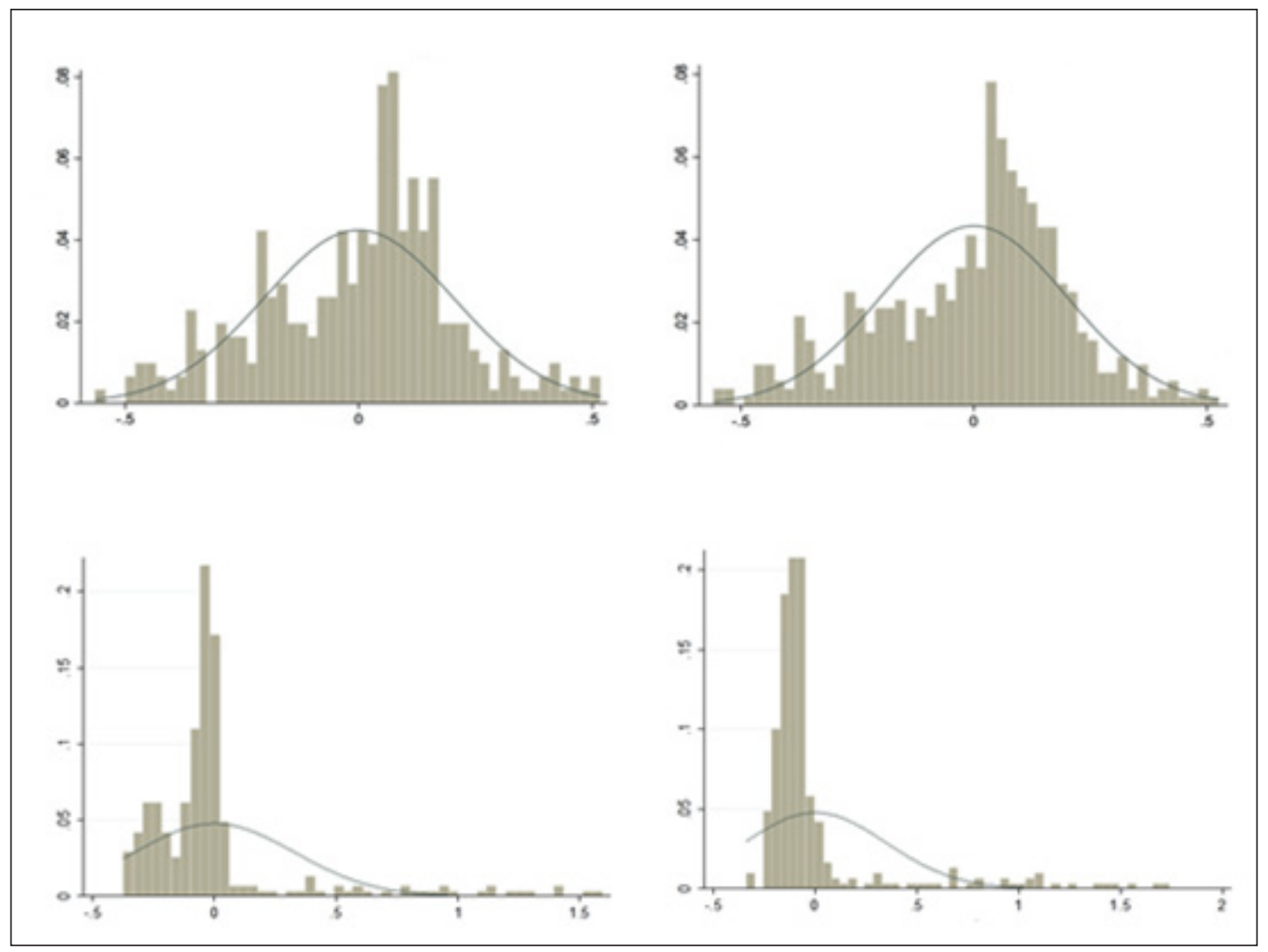

FIGURA 1 - Histograma dos resíduos.

FONTE: Elaborado pelos autores.

Numa observação dos histogramas da Figura 1, tem-se que os modelos "a" e "b" (análise 1) possuem distribuição dos resíduos assintoticamente normal. Porém, os modelos “c" e "d" (análise 2) não se aproximam de uma normalidade e, portanto, não são considerados. Dessa forma, passa-se a trabalhar apenas com os modelos da análise 1, ou seja, em que Y é a razão entre custos (CPV) e receitas (RLV).

Outras características dos testes de pressupostos da Tabela 2 são a heterocedasticidade e a autocorrelação em ambos os modelos $(a, b)$. Assim, para torná-los homocedásticos, utiliza-se o método de erros-padrões consistentes para heterocedasticidade de White, também conhecido como erro-padrão robusto. E, na presença de problemas de autocorrelação, utiliza-se a transformação do modelo original para o modelo de mínimos quadrados generalizados (MQG), por meio do método de Prais Winsten para regressão linear generalizada (Gujarati, 2006). Outros autores que, ao detectar a presença de autocorrelação em seus modelos, utilizaram o método MQG são Soares \& Kloeckner (2005), Dias et al. (2009) e Silva Jr. \& Bressan (2014), porém em outras áreas do conhecimento. Após a realização dos testes e correções supracitados, estimam-se os modelos de regressão para dados em painel, conforme as Tabelas 3 e 4 . 
TABELA 3 - Resultado da regressão da análise 1, modelo "a"

\begin{tabular}{|c|c|c|c|c|}
\hline & Coeficiente & Erro-padrão & $\mathbf{T}$ & $\mathbf{P}>|\mathbf{t}|$ \\
\hline Classe 2 & 0,000905 & 0,024470 & 0,04 & 0,971 \\
\hline Classe 3 & 0,080695 & 0,042938 & 1,88 & 0,061 \\
\hline Porte 2 & 0,004650 & 0,051773 & 0,09 & 0,928 \\
\hline Porte 3 & $-0,020656$ & 0,055341 & $-0,37$ & 0,709 \\
\hline Porte 4 & $-0,037208$ & 0,065439 & $-0,57$ & 0,570 \\
\hline Constante & 0,655949 & 0,062933 & 10,42 & 0,000 \\
\hline $\mathrm{N}^{0}$ de observações & 308 & \multicolumn{2}{|c|}{$\mathbf{R}^{2}$ ajustado } & 0,3059 \\
\hline Estatística F $(5,302)$ & 1,97 & \multicolumn{2}{|c|}{ Prob > F } & 0,0825 \\
\hline Estatística Durbin-Watson & 1,893740 & \multicolumn{2}{|c|}{ rho } & 0,8930638 \\
\hline
\end{tabular}

FONTE: elaborada pelos autores.

TABELA 4 - Resultado da regressão da análise 1, modelo "b"

\begin{tabular}{|c|c|c|c|c|}
\hline & Coeficiente & Erro-padrão & $\mathbf{T}$ & $\mathbf{P}>|\mathbf{t}|$ \\
\hline ISO 14001 & $-0,012569$ & 0,021729 & $-0,58$ & 0,563 \\
\hline Lei $n^{\circ} 9.991 / 2000$ & $-0,008819$ & 0,036961 & $-0,24$ & 0,811 \\
\hline Porte 2 & 0,044218 & 0,039582 & 1,12 & 0,264 \\
\hline Porte 3 & 0,033503 & 0,041870 & 0,80 & 0,424 \\
\hline Porte 4 & 0,043542 & 0,046906 & 0,93 & 0,354 \\
\hline Constante & 0,650370 & 0,054709 & 11,89 & 0,000 \\
\hline$N^{0}$ de observações & 512 & \multicolumn{2}{|c|}{$\mathbf{R}^{2}$ ajustado } & 0,2954 \\
\hline Estatística F $(2,506)$ & 0,59 & \multicolumn{2}{|c|}{ Prob $>$ F } & 0,7105 \\
\hline Estatística Durbin-Watson & 1,973934 & \multicolumn{2}{|c|}{ rho } & 0,8642947 \\
\hline
\end{tabular}

FONTE: Elaborada pelos autores.

Os resultados das Tabelas 3 e 4 utilizam erros-padrões robustos para correção de heterocedasticidade, obtendo-se um resultado ajustado de 0,893 para o modelo "a" e de 0,864 para o modelo "b", ou seja, passa-se a não rejeitar mais a hipótese nula de homocedasticidade. As estimações dos modelos também são realizadas com base em MQG e, com a correção de Durbin-Watson, os resíduos passam a não admitir mais autocorrelação, uma vez que, para a análise "a", o valor ajustado é de 1,893 e, para a análise "b", é de 1,973, os quais se enquadram na faixa de inexistência de autocorrelação da Tabela de Distribuição de Durbin-Watson (1,820 a 2,281).
Quanto à significância dos modelos, percebe-se que o modelo "a" mostrar-se-ia significante apenas se fosse considerado um nível de 90\% de intervalo de confiança, mas não ao nível usual de 95\%. Já o modelo "b" demonstra-se insignificante, haja vista que o Prob $>F$ é de 0,7105 , ou seja, o modelo teria um grau de confiança de aproximadamente 29\%. Ainda, em relação às Tabelas 3 e 4, o resultado do coeficiente de determinação $\left(\mathrm{R}^{2}\right)$ indica que na análise "a" as variáveis ISO 14001 e Carteira ISE, representadas em diferentes níveis de adoção (classes) das práticas sustentáveis, bem como a variável de controle porte, explicam conjun- 
tamente $30,59 \%$ da relação entre custos e receitas. Já no modelo "b", as variáveis ISO 14001 e Lei no $9.991 / 2000$ e a variável de controle porte explicam 29,54\% do comportamento dos custos.

Contudo, observa-se que todas as variáveis explicativas, para ambos os modelos, quando analisadas isoladamente, mostram-se não significantes a um nível usual de 95\% de confiança e, dessa forma, não é possível inferir estatisticamente que elas impactam a eficiência dos custos (CPV/RLV) do setor em análise.

Essa não significância pode ser derivada da proporcionalidade entre os custos e as receitas, o que faria com que seus efeitos individuais não fossem captados, devido à variável dependente ser composta da divisão entre esses valores. Assim, com no intuito de verificar essa possibilidade, realizam-se as análises isoladas de custos e de receitas, mediante os mesmos modelos de regressão e diretrizes anteriores, com o diferencial da transformação dos valores na função logarítmica, conforme empregado por outros estudos, como o de Ferron et al. (2012) e de Zaro et al. (2015). Salienta-se também que são realizadas novas exclusões de da- dos discrepantes, haja vista que estes podem variar por referir-se a valores integrais e não mais a uma divisão. Assim, tem-se um total de 841 observações para as análises de custos em ambos os modelos e 957 observações para as análises de receitas.

Quanto aos testes de pressupostos básicos, tanto para os dados de custo quanto para os de receita, em ambos os modelos, há presença de autocorrelação, heterocedasticidade e não normalidade dos resíduos. Portanto, utilizam-se os mesmos métodos de correção e modelos anteriormente citados. Dessa forma, têm-se os seguintes resultados das estimações dos modelos (Tabelas 5 e 6):

Conforme se pode perceber, todos os modelos apresentam-se significantes ao nível usual de 95\% de confiança. Os coeficientes de determinação $\left(\mathrm{R}^{2}\right)$ indicam que para o modelo "a" as variáveis independentes explicam $69,35 \%$ dos custos e 58,62\% das receitas. Já no modelo "b”, as variáveis independentes explicam $66,86 \%$ dos custos e $54,57 \%$ das receitas. Salienta-se que, em ambas as estimações, os valores ajustados passam a não admitir mais heterocedasticidade e autocorrelação, e a distribuição dos resíduos pode ser considerada normal em função da normalidade assintótica.

TABELA 5 - Resultado das regressões - Modelo "a"

\begin{tabular}{|c|c|c|c|c|c|c|c|}
\hline \multicolumn{4}{|c|}{ Variável dependente $=$ custos } & \multicolumn{4}{|c|}{ Variável dependente $=$ receitas } \\
\hline & Coeficiente & Erro-padrão & $\mathbf{P}>|\mathbf{t}|$ & & Coeficiente & Erro-padrão & $\mathbf{P}>|\mathbf{t}|$ \\
\hline Classe 2 & 0,1114691 & 0,0703050 & 0,114 & Classe 2 & 0,0355249 & 0,0516555 & 0,492 \\
\hline Porte 2 & 0,5060589 & 0,4041864 & 0,211 & Porte 2 & 0,5125359 & 0,2485544 & 0,040 \\
\hline Porte 3 & 0,5497493 & 0,3926698 & 0,162 & Porte 3 & 0,5449270 & 0,2457812 & 0,027 \\
\hline Porte 4 & 0,5877614 & 0,3841090 & 0,127 & Porte 4 & 0,5092676 & 0,2546092 & 0,046 \\
\hline \multicolumn{2}{|c|}{$N^{0}$ de observações } & \multicolumn{2}{|c|}{324} & \multicolumn{2}{|c|}{$\mathrm{N}^{0}$ de observações } & \multicolumn{2}{|c|}{354} \\
\hline \multicolumn{2}{|c|}{ Estatística F $(5,318)$} & \multicolumn{2}{|c|}{2,46} & \multicolumn{2}{|c|}{ Estatística F $(5,348)$} & \multicolumn{2}{|c|}{2,35} \\
\hline \multicolumn{2}{|c|}{$R^{2}$ ajustado } & \multicolumn{2}{|c|}{0,6935} & \multicolumn{2}{|c|}{$\mathbf{R}^{2}$ ajustado } & \multicolumn{2}{|c|}{0,5862} \\
\hline \multicolumn{2}{|l|}{ Prob $>$ F } & \multicolumn{2}{|c|}{$\mathbf{0 , 0 3 3 4}$} & \multicolumn{2}{|l|}{ Prob $>$ F } & \multicolumn{2}{|c|}{0,0405} \\
\hline
\end{tabular}

FONTE: elaborada pelos autores. 
TABELA 6 - Resultado das regressões - Modelo "b"

\begin{tabular}{|c|c|c|c|c|c|c|c|}
\hline \multicolumn{4}{|c|}{ Variável dependente $=$ custos } & \multicolumn{4}{|c|}{ Variável dependente $=$ receitas } \\
\hline & Coeficiente & Erro-padrão & $\mathbf{P}>|\mathbf{t}|$ & & Coeficiente & Erro-padrão & $\mathbf{P}>|\mathbf{t}|$ \\
\hline ISO 14001 & $-0,0457611$ & 0,1248113 & 0,714 & ISO 14001 & $-0,1282244$ & 0,1288176 & 0,320 \\
\hline Porte 2 & 0,4556479 & 0,2856237 & 0,111 & Porte 2 & 0,2848238 & 0,1207284 & 0,019 \\
\hline Porte 3 & 0,5341455 & 0,2790882 & 0,056 & Porte 3 & 0,3560515 & 0,1215701 & 0,004 \\
\hline Porte 4 & 0,6101138 & 0,2744579 & 0,027 & Porte 4 & 0,3932279 & 0,1267092 & 0,002 \\
\hline \multicolumn{2}{|c|}{$N^{0}$ de observações } & \multicolumn{2}{|c|}{$\mathbf{5 1 7}$} & \multicolumn{2}{|c|}{$\mathrm{N}^{0}$ de observações } & \multicolumn{2}{|c|}{603} \\
\hline \multicolumn{2}{|c|}{ Estatística F $(5,318)$} & \multicolumn{2}{|c|}{3,34} & \multicolumn{2}{|c|}{ Estatística F $(\mathbf{5}, \mathbf{5 9 7})$} & \multicolumn{2}{|c|}{4,24} \\
\hline \multicolumn{2}{|l|}{$\mathbf{R}^{2}$ ajustado } & \multicolumn{2}{|c|}{0,6686} & \multicolumn{2}{|c|}{$\mathbf{R}^{2}$ ajustado } & \multicolumn{2}{|c|}{$\mathbf{0 , 5 4 5 7}$} \\
\hline \multicolumn{2}{|l|}{ Prob $>$ F } & \multicolumn{2}{|c|}{0,0056} & \multicolumn{2}{|l|}{ Prob $>$ F } & \multicolumn{2}{|c|}{0,0008} \\
\hline
\end{tabular}

FONTE: elaborada pelos autores.

Ao analisar os resultados de custos, tem-se que as variáveis ISO 14001 e Lei no 9.991/2000, isoladamente, não apresentam significância estatística que possibilite afirmar que impactam os custos do período em análise. Entretanto, quando se analisam os diferentes níveis de adoção das práticas de GSCM (classes), ao tomar por base essa mesma certificação e a listagem da carteira ISE, percebe-se que, quanto mais elevado é esse nível, maiores são os custos organizacionais. Destaca-se que, apesar de a variável classe, em seu segundo nível, não ter mostrado significância, a um nível usual de $95 \%$ de confiança, esta não é desconsiderada, por tratar-se de uma análise conjunta.

Para as receitas, as variáveis ISO 14001 e Lei $n^{\circ}$ 9991/2000, isoladamente, bem como as variáveis classe, em todos os seus níveis, apresentam-se não significantes, ao nível usual de $95 \%$ de confiança. Dessa forma, não é possível fazer nenhum tipo de inferência sobre o impacto, no período em análise, dessas variáveis nas receitas das empresas do setor energético listadas na BM\&FBOVESPA.

Quanto à variável de controle porte, para as análises de receitas, em ambos os modelos, ela mostra-se significativa a um nível usual de 95\% de confiança e indica que, quanto maior é o porte da empresa (ativo total), maiores são as suas receitas. Já para as análises de custo, no modelo “b”, o porte em seu segundo nível não apresentou significância, mas continua a ser considerado em função de tratar-se de uma análise conjunta e também indica que, para níveis superiores, acrescem-se os custos. Entretanto, para o modelo "a", nenhum nível da variável porte mostrou-se significativo.

\section{Considerações finais}

Devido à crescente preocupação com a inclusão de práticas sustentáveis nas perspectivas organizacionais, o tema tem ganhado espaço nas discussões direcionadas ao comportamento dos custos, uma vez que o conhecimento quanto aos impactos financeiros dessas ações pode se tornar um fator relevante para o controle e o planejamento estratégico. Nesse contexto, tem-se a Green Supply Chain Management (GSCM) como uma abordagem utilizada para designar a incorporação de práticas 
sustentáveis, desde a extração das matérias-primas até a destinação final dos produtos e, portanto, busca-se compreender melhor o seu impacto sobre a eficiência dos custos organizacionais.

Isso posto, com base na literatura revisada, identificou-se como possíveis indicadores da influência das práticas da GSCM no comportamento dos custos a certificação ISO 14001, a listagem do Índice de Sustentabilidade Empresarial (ISE) e a Lei ${ }^{\circ}$ 9.991/2000.

Em resposta aos objetivos deste estudo, os resultados alcançados podem ser descritos em duas etapas. A primeira envolve o tratamento dos dados e os testes de adequação do modelo. Destaca-se que todos os pressupostos foram alcançados, bem como utilizaram-se os modelos adequados aos ajustes necessários. Quanto à segunda etapa, ou seja, a estatística aplicada aos dados, os resultados não podem inferir que houve variação na eficiência dos custos das empresas, com aumento ou diminuição da proporção de CPV sobre as RLV, uma vez que o efeito das práticas de GSCM em função das proxies utilizadas não se apresentou estatisticamente significante nas estimações, ao considerar os níveis usuais de intervalo de confiança.

Essas evidências se tornam antagônicas em relação às alegações de diminuição dos resultados financeiros, por meio da elevação dos custos, bem como aos relatos de elevação dos resultados, com redução de custos ou aumento das receitas, conforme proposto por autores referenciados nas seções anteriores, como, por exemplo, Raines (2002), Campos et al. (2009), Yang et al. (2010), Luthra et al. (2011), Ferron et al. (2012) e Zaro et al. (2015).

Mediante essa não significância e devido ao fato de que diversas variáveis podem ter afetado essa relação, em uma forma alternativa, analisam-se separadamente, como variáveis dependentes, os custos e as receitas, a fim de se obterem maiores indícios e esclarecimentos de seus comportamentos. Dessa forma, constatou-se que, de fato, os custos dos produtos vendidos sofrem elevação mediante maiores níveis de adequação às práticas de GSCM (classes), que utilizam como base a certificação ISO 14001 e a listagem da carteira ISE. Entretanto, no modelo "b", que analisa as variáveis ISO 14001 e Lei $n^{\circ}$ 9.991/2000 separadamente, estas não se apresentam significativas para explicar os custos organizacionais. Em relação às receitas, nenhuma das variáveis explicativas mostrou-se estatisticamente significante em ambos os modelos e, portanto, também não é possível fazer inferências sobre seus impactos. Também se constatou que, em todas as análises alternativas, cujas variáveis de controle porte mostraram-se significativas, quanto maior é o ativo da empresa (porte), maiores serão seus custos e receitas.

Contudo, quanto aos impactos de práticas de GSCM na eficiência produtiva das empresas, tem-se uma perspectiva diferenciada que foge do escopo até então seguido, pois, ao se analisar o setor energético, cuja regulamentação ambiental no Brasil é considerável, observa-se que, por mais que ocorram elevações nos custos, quando estes são analisados em relação às receitas auferidas, como forma de verificar sua eficiência, os resultados não se mostram significativos.

Cabe salientar que este estudo não afirma que as receitas ou que a medida de eficiência de custos (CPV/RLV) não sofrem impacto pela GSCM. Apenas não há possibilidade estatística de realizar afirmações nesse sentido, devido à não significância das variáveis.

O resultado de elevação dos custos, tomado isoladamente, fornece indícios de que a decisão pela adoção da GSCM pode ser motivada por outros fatores, que não apenas econômicos e financeiros, haja vista que as discussões quanto ao desempenho 
ambiental das organizações envolvem, além dessas determinantes, outros fatores que não são escopo deste estudo, cujos reflexos derivam de diferentes abordagens de cunho social e comportamental. Assim, mesmo que as empresas não obtenham resultados financeiros favoráveis, podem manter as práticas de GSCM devido a questões de imagem, obrigatoriedade legal ou pela sensibilização e preocupação socioambiental.

Dentre as limitações desta pesquisa, pode-se destacar a data de certificação ISO 14001, uma vez que esta é concedida por unidade da empresa e utilizou-se como referência a primeira certificação apresentada nos relatórios da administração, indiferente se o sistema de gestão ambiental se estende a todas as unidades ou parcialmente. Também destaca-se que a não significância estatística das variáveis pode ter sido influenciada pelo período anterior à certificação ISO 14001, em que as organizações introduziram práticas sustentáveis com o intuito de adequar-se às atribuições e exigências da certificação. Porém, como se tomou por base a data de certificação de fato, tais influências podem não ter sido captadas pelas análises. Essa afirmativa vai de encontro à alegação de Lamming \& Hampson (1996), que afirmam que os

\section{Referências}

Abbasi, M.; Nilsson, F. Themes and Challenges in making Supply Chains Environmentally Sustainable. Supply Chain Management: An International Journal, 17(5), 517-530, 2012. doi: $10.1108 / 13598541211258582$

Alberton, A.; Costa Jr., N. C. A. Meio ambiente e desempenho econômico-financeiro: benefícios dos Sistemas de Gestão Ambiental (SGAs) e o impacto da ISO 14001 nas Empresas Brasileiras. RAC-Eletrônica, 1(2), 153-171, 2007. Disponível em: <http://www.anpad.org.br/diversos/ trabalhos/EnANPAD/enanpad_2004/GSA/2004_GSA2596. pdf $>$. maiores custos organizacionais ocorrem no momento da implantação dos sistemas de gestão sustentável, devido aos investimentos realizados.

Por fim, ressalta-se que, neste artigo, os resultados dizem respeito às empresas do segmento de energia elétrica, durante o período de 1995 a 2014, listadas na BM\&FBOVESPA, devido à obrigatoriedade de publicação de seus demonstrativos financeiros, o que não permite generalizações. No entanto, para estudos futuros, podem-se desenvolver pesquisas em diferentes setores e não apenas em empresas de capital aberto, desde que se tenha acesso aos dados. Outra possibilidade para análises futuras diz respeito aos fatores que venham a esclarecer as fontes e o tempo de retorno dos investimentos ambientais, bem como a inclusão de perspectivas sociais e comportamentais associadas às econômicas.

Isso porque, por mais que este estudo trate apenas do setor energético, há particularidades e critérios específicos de cada empresa. Alguns, inclusive, são de natureza subjetiva, não captados pelo modelo apresentado, sendo necessárias análises mais detalhadas que busquem explicar essas peculiaridades.

Ambec, S.; Lanoie, P. Does It Pay to Be Green? A Systematic Overview. Academy of Management Perspectives, 22(4), 45-62, 2008. doi: 10.5465/AMP.2008.35590353

Ann, G. E.; Zailani, S.; Wahid, N. A. A study on the impact of environmental management system (EMS) certification towards firms' performance in Malaysia. Management of Environmental Quality: An International Journal, 17(1), 73-93, 2006. doi: 10.1108/14777830610639459

Arantes, A. F.; Jabbour, A. B. L. de S.; Jabbour, C. J. C. Adoção de práticas de Green Supply Chain Management: mecanismos de indução e a importância das empresas 
focais. Production, 24(4), 725-734, 2014. Disponível em: $<$ http://www.scielo.br/pdf/prod/2014nahead/a128813.pdf >

Babakri, K. A.; Bennett, R. A.; Franchetti, M. Critical factors for implementing ISO 14001 standard in United States industrial companies. Journal of Cleaner Production, 11(7), 749-752, 2003. doi: 10.1016/S0959-6526(02)00146-4

Barbieri, J. C. Competitividade internacional e normalização ambiental. Revista de Administração Pública RAP, 32(1), 57-71, 1998. Disponível em: <http://bibliotecadigital. fgv.br/ojs/index.php/rap/article/viewArticle/7686>.

BM\&FBOVESPA. $10^{a}$ Carteira de Índice de Sustentabilidade Empresarial - ISE, 2014. Disponível em: <http:// bmfbovespa.com.br/pt-br/a-bmfbovespa/sala-de-imprensa/ Releases/2014/BMFBOVESPA-divulga-a-10a-carteira-do-Indice-de-Sustentabilidade-Empresarial-2014-11-26. aspx?tipoNoticia $=32 \&$ idioma $=$ pt-br $>$. 2014. Acesso em: jan. 2016.

BM\&FBOVESPA. Índice de Sustentabilidade Empresarial - ISE. Disponível em: <http://bmfbovespa.com.br/indices/ ResumoIndice.aspx?Indice=ISE\&idioma=pt-br $>$. Acesso em: jan. 2016.

Brasil. Lei no 9.991, de 24 de Julho de 2000. Diário Oficial [da] República Federativa do Brasil. Poder Executivo, Brasília, DF, 2000.

Campos, L. M. S.; Grzebieluckas, C.; Selig, P. M. As empresas com certificação ISO 14001 são mais rentáveis? Uma abordagem em companhias abertas no Brasil. Revista Eletrônica de Administração, 15(1), 2009. Disponível em: $<$ http://www.lume.ufrgs.br/handle/10183/20752>.

Chien, M. K.; Shih, L. H. An empirical study of the implementation of green supply chain management practices in the electrical and electronic industry and their relation to organizational performances. International Journal of Environmental Science and Technology, 4(3), 383-394, 2007. Disponível em: $<$ http://www.bioline.org.br/pdf?st07049>.

Chunguang, Q.; Xiaojuan, C; Kexi, W.; Pan, P. Research on green logistics and sustainable development. In: International Conference on Information Management, Innovation Management and Industrial Engineering, ICIII'08. 2008.

Da Costa, F. M. G.; Voese, S. B.; Rosa, L. Custos e investimentos ambientais praticados pelas empresas do setor de energia elétrica que participam do ISE Bovespa
2008/2009. Revista Contabilidade e Controladoria, 1(3), 2009. doi: 10.5380/rcc.v1i3.20666

Dias, J.; Dias, M. H. A.; Lima, F. F. Os Efeitos da Política Educacional no Crescimento Econômico: Teoria e Estimativas Dinâmicas em Painel de Dados. Revista de Economia Política, 29(3), 232-251, 2009. Disponível em: <http:// www.rep.org.br/PDF/115-13.PDF>.

Fávero, L. P. Análise de dados. Rio de Janeiro: Elsevier: 2015 .

Ferron, R. T.; Funchal, B.; Nossa, V.; Teixeira, A. J. C. ISO 14001 Certification Effective? An Experimental Analysis of Firm Profitability. Brazilian Administration Review, 9, Especial, 78-94, 2012. doi: 10.1590/S1807-76922012000500006

Gujarati, D. Econometria Básica. 4. ed. Rio de Janeiro: Elsevier, 2006.

ISO - International Organization for Standardization. ISO 14000: Environment management. Disponível em: $<$ http:// www.iso.org/iso/home/standards/management-standards/ iso14000.htm>. Acesso em: jan. 2016.

Jong, P.; Paulraj, A.; Blome, C. The Financial Impact of ISO 14001 Certification: Top-Line, Bottom-Line, or Both? Journal of Business Ethics, 119(1), 131-149, 2014. doi: 10.1007/s10551-012-1604-Z

Lamming, R.; Hampson, J. The Environment as a Supply Chain Management Issue. British Journal of Management, 7(1), 45-62, 1996. doi: 10.1111/j.1467-8551.1996. tb00147.x

Lopes, L. J.; Neto, M. S.; Spers, V. R. E. Diferenças e complementaridades entre a logística reversa, ISO 14000 e o Green Supply Chain Management. Revista Gestão Industrial, 9(1), 225-253, 2013. Disponível em: <https:// portaldeinformacao.utfpr.edu.br/Record/peri-article-1056>.

Luthra, S.; Kumar, V.; Kumar, S.; Haleem, A. H. Barriers to implement green supply chain management in automobile industry using interpretive structural modeling techniqueAn Indian perspective. Journal of Industrial Engineering and Management, 4(2), 231-257, 2011. Disponível em: $<$ http://jiem.org/index.php/jiem/article/view/244>.

Marcondes, A. W.; Bacarji, C. D. ISE-Sustentabilidade no Mercado de Capitais. BM\&FBOVESPA. São Paulo: Report Editora. 2010. Disponível em: $<$ http://bmfbovespa.com.br/ 
pt-br/a-bmfbovespa/download/Livro-ISE.pdf > . Acesso em: jan. 2016.

Melvin, N. A method for the comparative analysis of the instructional costs of three baccalaureate nursing programs. Journal of Professional Nursing, 4(4), 249-261, 1988. doi:10.1016/S8755-7223(88)80010-3

Oliveira, O. J. de; Serra, J. R. Benefícios e dificuldades da gestão ambiental com base na ISO $14001 \mathrm{em}$ empresas industriais de São Paulo. Produção, 20(3), 429438, 2010. Disponível em: <http://www.scielo.br/pdf/ prod/2010nahead/aop_T6_0009_0078.pdf>.

ONU - Organizações das Nações Unidas. Relatório da comissão mundial sobre meio ambiente e desenvolvimento: Nosso Futuro Comum. 2. ed. São Paulo: FGV, 1991. Disponível em: <http://pt.scribd.com/doc/12906958/ Relatorio-Brundtland-Nosso-Futuro-Comum-Em-Portugues\#scribd>. Acesso em: jan. 2016.

Pak, S. A Review of the Literature and a Framework for Green Supply Chain Management. In: International Conference on Business, Economics, and Accounting. Bangkok: IBEA, 2013.

Porter, M. E.; Van der Lind, E, C. Toward a New Conception of the Environment-Competitiviness Relationship? Journal of Economic Perspectives, 9(4), 97-118, 1995.

Raines, S. S. Implementing ISO 14001 - An International Survey Assessing the Benefits of Certification. Corporate Environmental Strategy, 9(4), 418-426, 2002. doi: 10.1016/ S1066-7938(02)00009-X

Rao, P; Holt, D. Do green supply chains lead to competitiveness and economic performance? International Journal of Operations \& Production Management, 25(9), 898-916, 2005. doi: $10.1108 / 01443570510613956$

Ribeiro, R. B.; Santos, E. L. Análise das Práticas Estratégicas da Logística Verde no Gerenciamento da Cadeia de Suprimentos. Revista de Administração da Fatea, 5(5), 20-40, 2013. Disponível em: <http://fatea.br/seer/index. $\mathrm{php} / \mathrm{raf} /$ article/viewArticle/604>.

Richartz, F. O comportamento dos Custos das Empresas Brasileiras Listadas na BM\&FBOVESPA entre 1994 e 2011. Dissertação (Mestrado em Contabilidade) - UFSC, 2013.

Rogers, D.; Tibben-Lembke, R. S. Going backwards: reverse logistics trends and practices. Pittsburgh: RLEC Press. 1999.
Rover, S.; Borba, J. A.; Borgert, A. Como as empresas classificadas no Índice de Sustentabilidade Empresarial (ISE) evidenciam os custos e investimentos ambientais. Revista deCustose@gronegócio online, 4(1),2008. Disponível em: $<$ http://www.custoseagronegocioonline.com.br/numero1v4/Custos\%20ambientais\%20e\%20agronegocio.pdf $>$.

Seman, N. A. A.; Zakuan, N.; Jusoh, A.; Arif, M. S. M. Green Supply Chain Management: a Review and research direction. International Journal of Managing Value and Supply Chains (IJMVSC), 3(1), 1-18, 2012. doi: 10.5121/ ijmvsc.2012.3101

Seroka-Stolka, O. The development of green logistics for implementation sustainable development strategy in companies. Procedia-Social and Behavioral Sciences, 151, 302-309, 2014. doi: 10.1016/j.sbspro.2014.10.028

Seuring, S.; Sarkis, J.; Muller, M.; Rao, P. Sustainability and supply chain management - an introduction to the special issue. Journal of Cleaner Production, 16(15), 1545-1551, 2008. doi: 10.1016/j.jclepro.2008.02.002

Shank, J. K.; Govindarajan, V. A revolução dos custos: como reinventar e redefinir sua estratégia de custos para vencer em mercados crescentemente competitivos. 4. ed. Rio de Janeiro: Campus, 1997.

Silva Jr., J. M.; Bressan, V. G. F. Gerenciamento de resultados em cooperativas no Brasil: avaliando o Icome Smoothing às filiadas do SICREDI. In: Congresso USP Contabilidade e Controladoria. São Paulo: Congresso USP, 2014.

Soares, R. O.; Kloeckner, G. O. Controle acionário definido e endividamento: uma abordagem multimétodo. In: Congresso USP Contabilidade e Controladoria. São Paulo: Congresso USP, 2005.

Srisorn; W. The Benefit of Green Logistics to Organization. International Journal of Social. Human Science and Engineering, 7(8), 1182-1185, 2013.

Srivastava, S. K. Green supply-chain management: A stateof-the-art literature Review. International Journal of Management Reviews, 9(1), 53-80, 2007. doi: 10.1111/j.14682370.2007.00202.x

Walley, N.; Whitehead, B. It's not easy being green. Harvard Business Review, 72, 46-52. 1994. 
Yang, C. L.; Lin, S. P.; Chan, Y. H.; Sheu, C. Mediated effect of environmental management on manufacturing competitiveness: An empirical study. International Journal of Production Economics, 123(1), 210-220, 2010. doi: 10.1016/j.ijpe.2009.08.017
Zaro, E. S.; Zaro, C. S.; Richartz, F.; Borgert, A.; Bellen, H. M. V. The Impact of ISO 14001 Certification on Cost Behavior of Petrochemical Companies Listed in the BM\&FBOVESPA Stock Exchange. Environmental Quality Management, 24(3), 57-70, 2015. doi: 10.1002/tqem.21391 